\title{
Fields of Discipline Construction
}

\author{
Yongyi Qin \\ Guangxi University of Science and Technology \\ Liuzhou, China 545006
}

\begin{abstract}
Discipline refers to a relatively independent, specialized system of scientific knowledge. The discipline is not only the core of the university organization, but also the important foundation of university development. Therefore, discipline construction is the core work of promoting the connotative development of the university and improving the school quality and efficiency. Discipline construction has both academic activities and policy activities, of which the essence is to promote knowledge innovation through the effective allocation of resources. It also has a unique construction category. Talent is the core element in discipline construction. Construction funds are necessary conditions for the development of university. The university needs to establish the necessary institutional framework to standardize and strengthen the discipline construction management, promote the construction of first-class disciplines and enhance the development level.
\end{abstract}

\section{Keywords-university; discipline; discipline construction}

\section{INTRODUCTION}

With the development of science and technology and demands of social and national development, modern university has been in the status of constantly being found and defined. A scholar pointed out that "Modern university system has not existed already, but has been constructing." [1], disciplines in modern universities are also the same. The discipline is the logical starting point of the university, the axis of the reform on university system, and the discipline construction is the prerequisite for the development of the university. Discipline construction involves a wide range of fields including academic activities and policy activities, and the multiple functions of universities and characteristics of disciplines activities together decide the unique construction field and basic categories of discipline construction. The benign interaction mechanism of human resources, development fund, and policy system is the core dynamic mechanism that promotes the sound operation and development of modern university. It is the important contents of promoting the construction of first-class university, and deepening governance reform of modern university to ensure the operation effectiveness, scientificity and sustainability of this dynamic mechanism.

This paper is supported by the basic ability project fund of young teachers in Guangxi colleges and universities (Serial number:2017KY0336), the research project of Guangxi science and technology association(Serial number: 2016-Z-33) and Guangxi university of science and technology 2017 annual philosophy social science cultivation project.

\section{DISCIPLINE AND ITS CHARACTERISTICS}

\section{A. The Meaning of Discipline}

Discipline is the basic concept of scientific knowledge classification, which is used to refer to a relatively independent, specialized scientific knowledge system. Since the European Renaissance, the natural sciences have differentiated from the philosophical and derived a large number of knowledge systems, which expedites the development of modern science and technology. Various specialized knowledge system such as mechanics, astronomy, mathematics, physics, biology, medicine, chemistry and other natural sciences as well as political science, economics, sociology, management and other humanities science disciplines gradually formed and developed rapidly.

With the continuous development of scientific social system, concept of discipline appears in the field of knowledge production and dissemination. Corresponding to the word "discipline" in English, people divide the meaning of "xueke (学科)" into three aspects, namely: Discipline is the classification of scientific knowledge and subjects to learn, with the characteristics of specialization and professionalization; discipline as a specialized knowledge system has the meaning of social organization; discipline as a learning subject of knowledge system has the function of mandatory training, regularizing and shaping to scholars. Corresponding to this three-tier meaning, scholars in China summarize its meaning as follows: discipline originally refers to the standardized and specialized knowledge system formed in a certain historical period; disciplines extended around the standardization and specialization of the knowledge system, Specialized knowledge of the production and reproduction of the platform to provide the academic organization; disciplines implied to achieve the knowledge of the specialized and standardized and to control the object of study and scholars to train and shape a combination of power technology.[2] Based on the reality of the actual situation, this definition makes a reasonable summary for the concept and meaning of the discipline completely.

\section{B. The Origin of the Discipline and University}

With the various disciplines becoming the foundation of the university, the basic functions of university gradually evolve into the dissemination and exploration of scientific knowledge, personnel training and social services. In the development wave of modern science and technology in the mid-17th century to the end of the 18 th century, spontaneous 
and scattered scientific research have possessed the trend of specialization, but mostly outside the university. A variety of specialized knowledge system has become systematic and developed rapidly, but generally it is difficult to enter the university. Introduction of scientific research at the University of Berlin in Germany in the 19th century is the beginning of the modern university. Since then, it made remarkable achievements, promoted the world's higher education to undergo a fundamental change, and deeply affected the scientific research activities. [3] Since then, scientific research has become the basic function of modern university, and discipline system in universities has constantly enriched and developed. In every historical stage of technological development, modern universities have corresponding disciplines. Since the middle of the 20th century, a new round of scientific and technological revolution has led the various disciplines to continuously differentiate and tend to integrate, so a large number of new disciplines emerged, and disciplines of modern university became increasingly prosperous with a huge discipline system. With the basic functions of disseminating and exploring scientific knowledge based on the basic purpose of cultivating talents and, disciplines has become the organizational foundation of modern university organization.

\section{The Characteristics of the Discipline}

Discipline is the basic unit of modern university to perform its functions. Under certain organizational mechanism, its core element is specialized knowledge, which is expressed as a specific "disciplinary organization" at the organizational level with two typical characteristics.

1) Knowledge system: The core element of the discipline is the systematic expertise, the basic function of disciplinary organization is to disseminate the disciplinary knowledge, and carry out scientific research to achieve the purpose of personnel training. A variety of disciplines in modern universities have the disciplinary features of certain study scope, research methods and disciplines traditions.

2) Discipline structure: In the individual range of modern universities, disciplines often show a certain degree of correlation. Some scholars call this phenomenon as the "disciplinary structure" of modern university [4], discipline structures of different schools also tend to be different. Because of the correlation and interoperability of the knowledge and research methods among various knowledge systems, different disciplines can play a certain role in promoting each other's development. Therefore, in order to better realize their basic functions, modern universities often pay attention to build their own disciplinary system in the process of the development of disciplines, to promote the discipline system as a whole to show a unique "disciplinary structure."

\section{FIELDS OF DISCIPLINE CONSTRUCTION}

Discipline is the logical starting point of the university, the basic unit of realization of personnel training, scientific research and social services and other functions and the core and foundation of modern university organization. Based on the discipline organization system, modern university performs the basic functions of personnel training, scientific research, social services, and heritage of culture. Therefore, disciplines construction is of great significance for the university, which can be said to be the premise of university development. It is because of this that contemporary China put forward the construction of first-class discipline from the national level to promote the action plan of construction of first-class university. [5] Based on the basic functions of the university, discipline construction centering on the human, financial and material resources of discipline carry out activities from the three aspects of discipline team, discipline base and disciplinary policy that maintains the favorable operation of discipline organization.

\section{A. Discipline Team}

The development of disciplines cannot be separated from the growth and development of academic team. In general, the professor team as the representative of highest academic level of the discipline organization is the natural subject that exercises the academic power, as well as the main force of discipline construction, and the most valuable human resources of the university. Today, every famous university has the research experts and research results in the field of first-class disciplines, which cultivate a large number of high-level talents for the society. The essence of school development is the development of disciplines strength, discipline team and capacity to contribute to the society. Development of disciplines can't be talked about without the discipline team and it is difficult to achieve the dream of the university.

\section{B. Discipline Base}

Discipline base is the support of discipline, as well as the basic conditions for the development of disciplines. The development of disciplines cannot be separated from the growth of disciplinary knowledge while scientific research needs to rely on the scientific research conditions of the discipline base, such as laboratories, libraries, and test sites. The construction of the discipline base is conducive to optimizing the allocation, intensive use of scientific and technological innovation resources, and attracting disciplines talents to gather in the innovation base, to form the agglomeration effect. Discipline base plays an irreplaceable role in information exchange of discipline talents, complementary of discipline, cooperation of production and research, innovation integration, creation of discipline innovation culture, improvement of scientific research performance, and promotion discipline development.

\section{Disciplinary Policy}

The establishment of the system is of great significance to the discipline. The construction of the discipline is restricted by the administrative power and academic power of the university. The effect of the discipline development is influenced by the effect of coordinated operation of the two powers. Therefore, the scientific and rational discipline policy system is the system guarantee coordinating academic 
power and administrative power as well as the inevitable condition of the sound development of discipline. Perfect system is the basic requirements that ensure the development of academic and disciplines. On the contrary, if there is no complete discipline system, the negative phenomena of absence or dislocation of subjects will appear in subject construction.

\section{CONCLUSION}

Discipline plays an important role in the university through institutionalization, and the discipline construction status and indicators is an important indicator reflecting the development level of a school both at home and abroad. In particular, the subject construction of the university is mainly carried out in the three aspects of discipline team, discipline base, and discipline policy including the construction of disciplinary orientation (subject level and development level), discipline team (academic leader and echelon), scientific research, personnel training, discipline base (laboratory, key disciplines and equipment, etc.) and discipline management. The effect of discipline construction has become the main basis for evaluating modern universities.

\section{REFERENCES}

[1] Wang Hongcai. On the Structural Characteristics of Modern University System [J]. Fudan Education Forum, 2006. (01).

[2] Hu Jianhua, Wang Jianhua, Wang Quanlin, et al. Discussion on the reform of university system [M]. Nanjing: Nan Jing Normal University Press, 2006.

[3] Hu Jianhua, Chen Lie, Zhou Chuan, et al. New theory of higher education [M]. Nanjing: Jiangsu Education press, 2006

[4] Pang Qingshan. Study on the Disciplinary Structure and Discipline System of Universities [D]. Shanghai: East China Normal University Doctoral Dissertation, 2004

[5] the State Council. Notice on Issuing and Distributing the Overall Plan for Promoting World-class Universities and First-class Discipline Construction [EB/OL]. http://www.gov.cn/zhengce/content/201511/05/content_10269.htm, 2015-11-05. 